\title{
The Stability of Cylindrical Shells Containing an FGM Layer Subjected to Axial Load on the Pasternak Foundation
}

\author{
${ }^{1}$ Abdullah Heydaroglu Sofiyev, ${ }^{2}$ Mehmet Avcar \\ ${ }^{1,2}$ Department of Civil Engineering of Suleyman Demirel University, Isparta, Turkey \\ E-mail:asofiyev@mmf.sdu.edu.tr,mavcar@mmf.sdu.edu.tr \\ Received July 7, 2009; revised November 4, 2009; accepted November 12, 2009
}

\begin{abstract}
In this study, the stability of cylindrical shells that composed of ceramic, FGM, and metal layers subjected to axial load and resting on Winkler-Pasternak foundations is investigated. Material properties of FGM layer are varied continuously in thickness direction according to a simple power distribution in terms of the ceramic and metal volume fractions. The modified Donnell type stability and compatibility equations on the Pasternak foundation are obtained. Applying Galerkin's method analytic solutions are obtained for the critical axial load of three-layered cylindrical shells containing an FGM layer with and without elastic foundation. The detailed parametric studies are carried out to study the influences of thickness variations of the FGM layer, radius-to-thickness ratio, material composition and material profile index, Winkler and Pasternak foundations on the critical axial load of three-layered cylindrical shells. Comparing results with those in the literature validates the present analysis.
\end{abstract}

Keywords: FGM Layer, Stability, Cylindrical Shell, Critical Axial Load, Winkler and Pasternak Foundations

\section{Introduction}

Functionally graded materials (FGM) are characterized by a gradual change in properties within the specimen as a function of the position coordinates. The property gradient in the material is typically caused by a position-dependent chemical composition, micro structure or atomic order. There are several studies about the processing of FGM, and an overview of the different manufacturing methods can be found in Koizumi [1] and Kieback et al. [2]. The mechanical behavior of FGMs shells, such as bending due to mechanical loads, free vibration, stability and buckling, etc., have been studied by many scientists [3-8]. However, the literature on the investigation of vibration and stability problems of composite structures that composed of ceramic, FGM, and metal layers is scarce. A self-consistent constitutive framework is proposed to describe the behaviour of a generic threelayered system containing an FGM layer subjected to thermal loading given by Pitakthapanaphong and Busso [9]. Na and Kim [10] investigated three dimensional thermo-mechanical buckling analysis for FG composite plates that composed of ceramic, FGM, and metal layers by using the finite element methods. Liew et al. [11] presented the non-linear vibration analysis for layered cylindrical panels containing FGMs and subjected to a temperature gradient arising from steady heat conduction through the panel thickness. Lia and Batra [12] investigated buckling of axially compressed thin cylindrical shells with the functionally graded middle layer. Sofiyev et al. [13] and Sofiyev [14] studied vibration and stability of three-layered truncated conical and cylindrical shells containing an FGM layer subjected to an axial compressive load, respectively.

For some cases, these shells are embedded in an elastic medium. There are different approaches to analyze the interaction between a structure and an ambient medium. Most earthen soils can be appropriately represented by a mathematical model from Pasternak, whereas sandy soils and liquids can be represented by Winkler's model [15].

Despite the evident importance of practical applications cylindrical shells, the investigations on vibration and stability of homogeneous and FGM shells on elastic foundations are limited in the literature [16-23]. The stability problem of composite cylindrical shells that composed of ceramic-FGM-metal layers embedded in an elastic medium, subjected to an axial compressive load 
have not been studied yet. In the present work, an attempt is made to address this problem. Therefore, it is very important to develop an accurate, reliable analysis towards the understanding of the stability characteristics of the layered FGM structures with the effect of an elastic medium.

\section{Formulation of the Problem}

Figure 1(a) shows a composite cylindrical shell with simply supported edge conditions, composed of ceramic, FGM, and metal layers, of length $L$, radius $R$ and total thickness $h=h_{1}+h_{2}$. The ceramic and metal layers are assumed to be homogeneous and isotropic. The shell is referred to a coordinate system $x y z$ in which $x$ and $y$ is in the axial and circumferential directions of the shell and $z$ is in the direction of the inward normal to the middle surface. The origin of the coordinate system is located at the end of the shell, on the reference surface of the FGM layer. The three layered cylindrical shell containing an FGM layer is subjected to an axial compressive load $T$ (Figure 4):

$$
N_{x}^{0}=-T, \quad N_{y}^{0}=0, \quad N_{x y}^{0}=0
$$

The in plane geometry of the layered structure is shown in Figure 1(b). The FGM layer extends from $z=-a$ to $z=+a$ and, for continuous property assumptions to be valid; the thickness of this layer must be significantly larger than dominant micro structural length scale (e.g. grain size). The interfaces between the different layers are assumed to be perfectly bonded at all times and the multilayer system behaviour to be linear elastic.

We assume that the composition is varied from the bottom to top surface, i.e. the bottom surface $(z=-a)$ of the layer is metal-rich whereas the top surface $(z=a)$ is ceramic-rich. In such a way, the effective material properties $P$, like Young's modulus $E$ or Poisson's ratio can be expressed as

$$
P=P_{c} V_{c}+P_{m} V_{m}
$$

in which $P_{c}$ and $P_{m}$ denote the temperature-dependent properties of the ceramic and metal surfaces of the FGM layer, respectively.

$V_{c}$ and $V_{m}$ are the ceramic and metal volume fractions of the FGM layer and are related by

$$
V_{c}+V_{m}=1
$$

Following Reddy and his co-workers, the ceramic volume fraction $V_{c}$ is assumed as

$$
V_{c}=\left(\frac{z+a}{2 a}\right)^{N}
$$

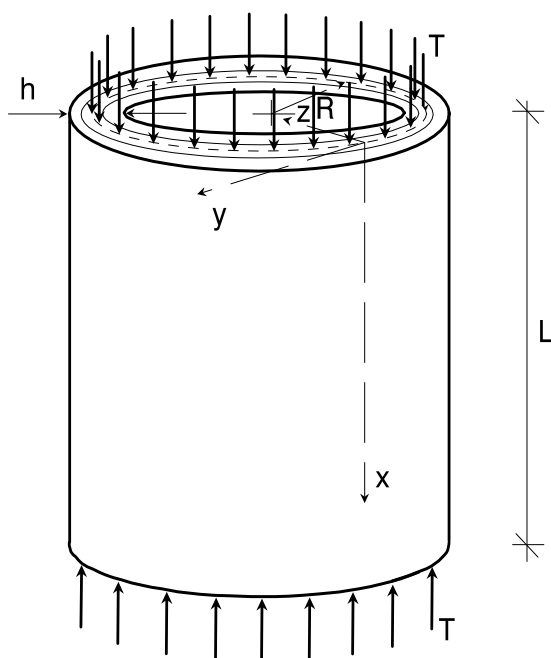

(a)

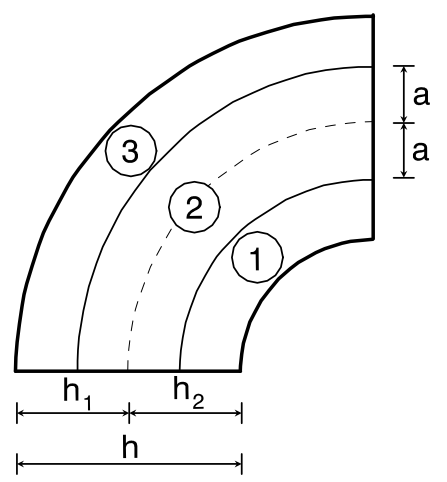

(b)

Figure 1. Schematic representation of (a) a three layered cylindrical shell and coordinate axes; (b) dimensions of the three-layered system.

where $N$ is the volume fraction index $0 \leq N<\infty$.

$V_{c}$ is satisfying the following conditions at homogeneous layers interfaces [9],

$$
V_{c}=\left\{\begin{array}{l}
0 \text { at } z=-a \\
1 \text { at } z=a
\end{array}\right.
$$

Variation of volume fraction $V_{c}$ in the thickness direction of composite cylindrical shell composed of Ceramic-FGMMetal layers is shown in Figure 2. The top surface is fully ceramic and bottom surface is fully metal of the FGM layer. The horizontal-axis stands for the volumetric percentage of ceramic while vertical-axis represents the position along the thickness of shell. Metal is the dominant constituent at the bottom layer and its volume fraction is increased continually from the bottom to the top of FGM layer. At the top layer, ceramic is the dominant constituent. 


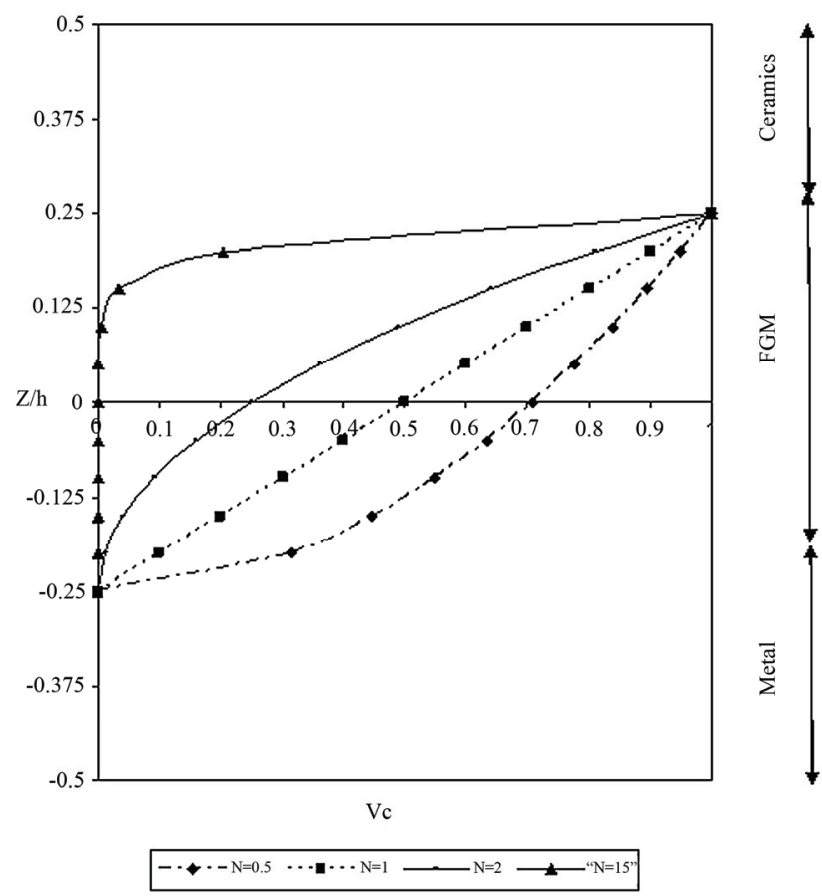

Figure 2. Variation of volume fraction $V_{c}$ in thickness direction of the Ceramic-FGM-Metal three layered shell.

From Equations (2)-(5), the Young's modulus $\hat{E}$ and the Poisson's ratio $\hat{v}$ of an FGM layer can be written as

$$
\hat{E}=\left(E_{c}-E_{m}\right) V_{c}+E_{m} ; \quad \hat{v}=\left(v_{c}-v_{m}\right) V_{c}+v_{m}
$$

where $E_{m}, v_{m}$ and $E_{c}, v_{c}$ are the Young's modulus and Poisson's ratio of the metal and ceramic surfaces of

$$
\begin{gathered}
E(z)=\left\{\begin{array}{l}
E_{0 m} \\
E_{m}+\left(E_{c}-E_{m}\right) V_{c} \\
E_{0 c}
\end{array}\right. \\
v(z)=\left\{\begin{array}{l}
v_{0 m} \\
v_{m}+\left(v_{c}-v_{m}\right) V_{c} \\
v_{0 c}
\end{array}\right.
\end{gathered}
$$

homogeneous metal and ceramic materials, respectively.

\section{Basic Equations}

The stress-strain relations for three layered shells containing an FGM layer are given as follows [11, 14]:

$$
\left\{\begin{array}{l}
\sigma_{x}^{(k)} \\
\sigma_{y}^{(k)} \\
\sigma_{x y}^{(k)}
\end{array}\right\}=\left[\begin{array}{ccc}
Q_{11}^{(k)} & Q_{12}^{(k)} & 0 \\
Q_{12}^{(k)} & Q_{11}^{(k)} & 0 \\
0 & 0 & Q_{66}^{(k)}
\end{array}\right]\left\{\begin{array}{l}
e_{x}-z \frac{\partial^{2} w}{\partial x^{2}} \\
e_{y}-z \frac{\partial^{2} w}{\partial y^{2}} \\
e_{x y}-z \frac{\partial^{2} w}{\partial x \partial y}
\end{array}\right\}
$$

the FGM layer, respectively.

In the majority of practical applications involving FGMs, they are used as interlayer between two homogeneous materials. For such cases, the through-thickness variation of the Young's modulus and Poisson's ratio in the three-layered system are (7.1) and (7.2). where $E_{0 m}, v_{0 m o}$ and $E_{0 c}, v_{0 c}$ are the Young's modulus and Poisson's ratio of the

$$
\begin{array}{cc}
\text { for } & -h_{1} \leq z \leq-a \\
\text { for } & -a \leq z \leq a \\
\text { for } & a \leq z \leq h_{2} \\
\text { for } & -h_{1} \leq z \leq-a \\
\text { for } & -a \leq z \leq a \\
\text { for } & a \leq z \leq h_{2}
\end{array}
$$$$
\text { for }
$$

where $\sigma_{x}^{(k)}, \sigma_{y}^{(k)}$ and $\sigma_{x y}^{(k)}$ are the stresses in the layers, $e_{x}$ and $e_{y}$ are the normal strains in the curvilinear coordinate directions $x$ and $y$ on the reference surface, respectively, whereas $e_{x y}$ is the corresponding shear strain; $w$ is the displacement of the reference surface in the normal direction, positive towards the axis of the cylinder and assumed to be much smaller than the thickness, a comma denotes partial differentiation with respect to the corresponding coordinates.

The quantities $Q_{i j}^{(k)}, \quad i, j=1,2,6 ; k=1,2,3$ for lamina are 


$$
\begin{gathered}
Q_{11}^{(1)}=Q_{22}^{(1)}=\frac{E_{0 m}}{1-v_{0 m}^{2}}, Q_{12}^{(1)}=\frac{v_{0 m} E_{0 m}}{1-v_{0 m}^{2}}, Q_{66}^{(1)}=\frac{E_{0 m}}{1+v_{0 m}} \\
Q_{11}^{(2)}=Q_{22}^{(2)}=\frac{\left(E_{c}-E_{m}\right) V_{c}+E_{m}}{1-\left[\left(v_{c}-v_{m}\right) V_{c}+v_{m}\right]^{2}} \\
Q_{12}^{(2)}=\frac{\left[\left(E_{c}-E_{m}\right) V_{c}+E_{m}\right]\left[\left(v_{c}-v_{m}\right) V_{c}+v_{m}\right]}{1-\left[\left(v_{c}-v_{m}\right) V_{c}+v_{m}\right]^{2}} \\
Q_{11}^{(3)}=Q_{22}^{(3)}=\frac{E_{0 c}}{1-v_{0 c}^{2}}, \quad Q_{12}^{(3)}=\frac{v_{0 c} E_{0 c}}{1-v_{0 c}^{2}}, Q_{66}^{(3)}=\frac{\left.E_{0 c}-E_{m}\right) V_{c}+E_{m}}{1+v_{0 c}}
\end{gathered}
$$

The well-known force and moment resultants are expressed by $[14,25]$

$$
\left[\left(N_{x}, N_{y}, N_{x y}\right),\left(M_{x}, M_{y}, M_{x y}\right)\right]=\sum_{k=1}^{3} \int_{z k}^{z k+1}(1, \mathrm{z})\left[\sigma_{x}^{(k)}, \sigma_{y}^{(k)}, \sigma_{x y}^{(k)}\right] \mathrm{d} z
$$

The relations between the forces $N_{x}, N_{y}, N_{x y}$ and the Airy stress function $\bar{\Phi}=\Phi / h$ are given by

$$
\left\{N_{x}, \mathrm{~N}_{y}, \mathrm{~N}_{x y}\right\}=\left\{\frac{\partial^{2} \Phi}{\partial y^{2}}, \frac{\partial^{2} \Phi}{\partial x^{2}},-\frac{\partial^{2} \Phi}{\partial x \partial y}\right\}
$$

For the elastic foundation, one assumes the two-parameter elastic foundation model proposed by Pasternak [15]. The foundation medium is assumed to be linear, homogenous and isotropic. The bonding between the truncated conical shell and the foundation is perfect and frictionless. If the effects of damping and inertia force in the foundation are neglected, the foundation interface pressure $\mathrm{p}$ may be expressed as

$$
p=K_{w} w-K_{p}\left(\frac{\partial^{2} w}{\partial x^{2}}+\frac{\partial^{2} w}{\partial y^{2}}\right)
$$

where $K_{w}$ is the modulus of subgrade reaction for the foundation and $K_{p}$ the shear modulus of the subgrade. Note that by setting $K_{p}=0$, the Pasternak model becomes that of the Winkler foundation model.

When the above assumptions are taken into consideration, the modified Donnell type stability and compatibility equations of composite cylindrical shells that composed of ceramic, FGM, and metal layers, subjected axial compressive load and resting on the Pasternak foundation are given, respectively by

$$
\begin{gathered}
\frac{\partial^{2} M_{x}}{\partial x^{2}}+2 \frac{\partial^{2} M_{x y}}{\partial x \partial y}+\frac{\partial^{2} M_{y}}{\partial y^{2}}+\frac{N_{y}}{R}-T \frac{\partial^{2} w}{\partial x^{2}} \\
-K_{w} w+K_{p}\left(\frac{\partial^{2} w}{\partial x^{2}}+\frac{\partial^{2} w}{\partial y^{2}}\right)=0
\end{gathered}
$$

$$
\frac{\partial^{2} e_{x}}{\partial y^{2}}+\frac{\partial^{2} e_{y}}{\partial x^{2}}-2 \frac{\partial^{2} e_{x y}}{\partial x \partial y}=-\frac{1}{R} \frac{\partial^{2} w}{\partial x^{2}}
$$

where $M_{x}, M_{x y}, M_{y}$ are moment resultants.

Substituting expressions (8)-(11) and (12) in Equations. (13) and (14) a system of differential equations for the stress function and the normal displacement can be obtained in form as

$$
\left[\begin{array}{ll}
L_{11} & \mathrm{~L}_{12} \\
L_{21} & \mathrm{~L}_{22}
\end{array}\right]\left[\begin{array}{l}
\Phi \\
w
\end{array}\right]=0
$$

where the following definitions apply:

$$
\begin{gathered}
L_{11}=A_{2}\left(\frac{\partial^{4}}{\partial x^{4}}+\frac{\partial^{4}}{\partial y^{4}}\right)+2\left(A_{1}-A_{5}\right) \frac{\partial^{4}}{\partial x^{2} \partial y^{2}}+\frac{1}{R} \frac{\partial^{2}}{\partial x^{2}} \\
L_{12}=-A_{3}\left(\frac{\partial^{4}}{\partial x^{4}}+\frac{\partial^{4}}{\partial y^{4}}\right)-2\left(A_{4}+A_{6}\right) \frac{\partial^{4}}{\partial x^{2} \partial y^{2}} \\
-T \frac{\partial^{2}}{\partial x^{2}}-K_{w}+K_{p}\left(\frac{\partial^{2}}{\partial x^{2}}+\frac{\partial^{2}}{\partial y^{2}}\right) \\
\mathrm{L}_{21}=\mathrm{B}_{1}\left(\frac{\partial^{4}}{\partial x^{4}}+\frac{\partial^{4}}{\partial y^{4}}\right)+2\left(B_{2}+B_{5}\right) \frac{\partial^{4}}{\partial x^{2} \partial y^{2}} \\
\mathrm{~L}_{22}=\frac{1}{\mathrm{R}} \frac{\partial^{2}}{\partial x^{2}}-B_{4}\left(\frac{\partial^{4}}{\partial x^{4}}+\frac{\partial^{4}}{\partial y^{4}}\right)-2\left(B_{3}+B_{6}\right) \frac{\partial^{4}}{\partial x^{2} \partial y^{2}}
\end{gathered}
$$

in which expressions $A_{i}, B_{i}(i=1-6)$ are defined as follows:

$$
\begin{gathered}
A_{1}=C_{11} B_{1}+C_{21} B_{2}, \\
A_{2}=C_{11} B_{2}+C_{21} B_{1}, \\
A_{3}=C_{11} B_{3}+C_{21} B_{4}+C_{12}, \\
A_{4}=C_{11} B_{4}+C_{21} B_{3}+C_{22}, \\
A_{5}=C_{61} B_{5}, \quad A_{6}=C_{61} B_{6}+C_{62}, \\
B_{1}=C_{10} D, \\
B_{2}=-C_{20} D, \\
B_{3}=\left(C_{20} C_{21}-C_{11} C_{10}\right) D, \\
B_{4}=\left(C_{20} C_{11}-C_{21} C_{10}\right) D, \\
B_{5}=1 / C_{60}, \\
B_{6}=C_{61} / C_{60}, \\
D=1 /\left[\left(C_{10}\right)^{2}-\left(C_{20}\right)^{2}\right]
\end{gathered}
$$


in which expressions $C_{1 k_{1}}, C_{2 k_{1}}$ and $C_{6 k_{1}}\left(k_{1}=0,1,2\right)$ are defined as follows:

$$
\begin{aligned}
& C_{1 k_{1}}=\frac{E_{0 m}}{1-v_{0 m}^{2}} \int_{-h_{1}}^{-a} z^{k 1} \mathrm{~d} z+ \\
& \int_{-a}^{a} z^{k 1} \frac{\left(E_{c}-E_{m}\right) V_{c}+E_{m}}{1-\left[\left(v_{c}-v_{m}\right) V_{c}+v_{m}\right]^{2}} \mathrm{~d} z+\frac{E_{0 c}}{1-v_{0 c}^{2}} \int_{a}^{h_{2}} z^{k 1} \mathrm{~d} z \\
& C_{2 k 1}=\int_{-a}^{a} z^{k_{1}} \frac{\left[\left(E_{c}-E_{m}\right) V_{c}+E_{m}\right]\left[\left(v_{c}-v_{m}\right) V_{c}+v_{m}\right]}{1-\left[\left(v_{c}-v_{m}\right) V_{c}+v_{m}\right]^{2}} \mathrm{~d} z+ \\
& +\frac{E_{0 m}}{1-v_{0 m}^{2}} \int_{-h_{1}}^{-a} z^{k_{1}} \mathrm{~d} z+\frac{E_{0 c}}{1-v_{0 c}^{2}} \int_{a}^{h_{2}} z^{k_{1}} \mathrm{~d} z \\
& C_{6 k 1}=\frac{E_{0 m}}{1+v_{0 m}} \int_{-h_{1}}^{-a} z^{k 1} \mathrm{~d} z+\int_{-a}^{a} z^{k_{1}} \frac{\left(E_{c}-E_{m}\right) V_{c}+E_{m}}{1+\left(v_{c}-v_{m}\right) V_{c}+v_{m}} \mathrm{~d} z \\
& +\frac{E_{0 c}}{1+v_{0 c}} \int_{a}^{h_{2}} z^{k 1} \mathrm{~d} z
\end{aligned}
$$

Equation (15) is the basic differential equations for the vibration and stability of generic composite cylindrical shells containing an FGM layer resting on a Pasternak foundation.

\section{Solution of Basic Equations}

Consider a cylindrical shell with simply supported edge conditions. The solution of the system of equation (15) is sought as follows [24]:

$$
\mathrm{w}=\xi \sin \frac{\mathrm{m}_{1} x}{R} \sin \frac{\mathrm{ny}}{\mathrm{R}}, \quad \Phi=\zeta \sin \frac{\mathrm{m}_{1} x}{R} \sin \frac{\mathrm{ny}}{\mathrm{R}}
$$

where $m_{1}=m \pi R / L, m$ is the half wave length in the direction of the x-axis, $n$ is the wave number in the direction of the $\mathrm{y}$-axis, $\xi$ and $\zeta$ are the amplitudes.

Substituting expression (19) in the equation set (15), applying Galerkin's method in the ranges $0 \leq x \leq L$ and $0 \leq y \leq 2 \pi R$ and eliminating $\zeta$ from the equations, the following equation for the critical axial load is obtained:

$$
\begin{aligned}
& T_{w p}^{c r}=\frac{1}{m_{1}^{2} R^{2}}\left\{\left[A_{3}\left(m_{1}^{4}+n^{4}\right)+2\left(A_{4}+A_{6}\right) m_{1}^{2} n^{2}\right]+\right. \\
& {\left[m_{1}^{2} R-A_{2}\left(m_{1}^{4}+n^{4}\right)-2\left(A_{1}-A_{5}\right) m_{1}^{2} n^{2}\right]} \\
& \left.\times \frac{\left[m_{1}^{2} R+B_{4}\left(m_{1}^{4}+n^{4}\right)+2\left(B_{3}+B_{6}\right) m_{1}^{2} n^{2}\right]}{B_{1}\left(m_{1}^{4}+n^{4}\right)+2\left(B_{2}+B_{5}\right) m_{1}^{2} n^{2}}\right\} \\
& +\frac{R^{2}\left[K_{w}+K_{p}\left(m_{1}^{2}+n^{2}\right) R^{-2}\right]}{m_{1}^{2}}
\end{aligned}
$$

The minimum values of the critical axial load $\left(T_{w p}^{c r}\right)$ are obtained by minimizing Equation (20) with respect to $\mathrm{m}$ and $\mathrm{n}$, the number of longitudinal and circumferential buckling waves.

As $K_{p}=0$, from Equation (20), in special case for the critical axial load $\left(T_{w}^{c r}\right)$ of three layered cylindrical shells containing an FGM layer on the Winkler foundation is obtained.

As $K_{w}=K_{p}=0$, from Equation (20), in special case for the critical axial load ( $T^{c r}$ ) of three layered cylindrical shells containing an FGM layer without an elastic foundation is obtained.

\section{Numerical Results and Discussions}

The buckling loads of single-layer orthotropic and $\left(0^{\circ} / 90^{\circ} / 0^{\circ}\right)$ cross-ply laminated graphite/epoxy circular cylindrical shells under pure axial load are compared in Table 1 with results of the Jones and Morgan [25], using their material properties, i.e., $E_{1}=30 \times 10^{6} \mathrm{psi} ; E_{2}=0.75 \times$ $10^{6} \mathrm{psi} ; G_{12}=0.375 \times 10^{6} \mathrm{psi} ; v_{1}=0.25 ; v_{2}=0.0625$, and cylindrical shell parameters $L=34.64$ in; $R=10.0 \mathrm{in} ; h=$ 0.12 in. It can be seen that the present results are in very good agreement with results of Jones and Morgan [25].

As there are presently no results in the open literature for the buckling of cylindrical shells under an axial load and resting on elastic foundations, comparison of results in this study is made with those of Paliwal et al. [17] for the free vibration analysis of homogenous isotropic cylindrical shells resting on a Winkler foundation. The comparison is shown in Table 2.

When the inertial term is added into the left side of Equation (13), after integrating and after some mathematical operations for the dimensionless frequency parame-

Table 1. Comparisons of dimensionless critical axial loads for single-layer orthotropic and $\left(0^{\circ} / 90^{\circ} / 0^{\circ}\right)$ cross-ply laminated cylindrical shells.

\begin{tabular}{ccc}
\hline & Jones and Morgan [25] & Present study \\
\hline Lay-up & $T^{c} L^{2} / E_{2} h^{3}$ & $T^{c r} L^{2} / E_{2} h^{3}$ \\
\hline$\left(0^{\circ}\right)$ & 1482 & $1482.0(3,7)$ \\
$\left(0^{\circ} / 90^{\circ} / 0^{\circ}\right)$ & 1859.8 & $1859.8(3,6)$ \\
\hline
\end{tabular}

Table 2. Comparison of the dimensionless frequency parameter $\omega_{1 \mathrm{w}}$ for a cylindrical shell resting on the Winkler foundation $\left(\mathrm{R} / \mathrm{h}=100 ; \mathrm{L} / \mathrm{R}=2 ; \mathrm{K}_{\mathrm{w}}=10^{-4} \mathrm{~N} / \mathrm{m}^{3}\right)$.

\begin{tabular}{cc}
\hline Paliwal et al. $[17]$ & Present study \\
\hline $0.6788(1)$ & $0.6792(1)$ \\
$0.3639(2)$ & $0.3646(2)$ \\
$0.2053(3)$ & $0.2080(3)$ \\
$0.1275(4)$ & $0.1382(4)$ \\
\hline
\end{tabular}


ter $w_{1 w}$ of the free vibration of FGM cylindrical shells resting on the Winkler-Pasternak foundations, the following expression is obtained in Equation (21):

where the mass density per unit length defined as

$$
\rho_{t}=\rho_{0 m}\left(h_{1}-a\right)+\int_{-\mathrm{a}}^{\mathrm{a}}\left[\left(\rho_{c}-\rho_{m}\right) V_{c}+\rho_{m}\right] \mathrm{d} z+\rho_{0 c}\left(h_{2}-a\right)
$$

This comparison is to ensure that the elastic foundation effects have been correctly integrated into the present formulation.
Based on the above comparisons of Tables $\mathbf{1}$ and $\mathbf{2}$, the accuracy of the present study is validated.

\subsection{Numerical Results}

The buckling analysis of three layered cylindrical shells containing an FGM layer was conducted for ceramic and metal combinations. The FGM material considered was Zirconium oxide and Titanium alloy, referred to as $\mathrm{ZrO}_{2} / \mathrm{Ti}-6 \mathrm{Al}-4 \mathrm{~V}$ or FGM. The temperature dependence

$$
\omega_{1 w}=\sqrt{\frac{1}{\rho_{t}}\left\{\begin{array}{l}
\frac{1}{R^{4}}\left\{\left[A_{3}\left(m_{1}^{4}+n^{4}\right)+2\left(A_{4}+A_{6}\right) m_{1}^{2} n^{2}\right]+\left[\begin{array}{l}
m_{1}^{2} R-A_{2}\left(m_{1}^{4}+n^{4}\right) \\
-2\left(A_{1}-A_{5}\right) m_{1}^{2} n^{2}
\end{array}\right]\right. \\
\left.\times \frac{\left[m_{1}^{2} R+B_{4}\left(m_{1}^{4}+n^{4}\right)+2\left(B_{3}+B_{6}\right) m_{1}^{2} n^{2}\right]}{B_{1}\left(m_{1}^{4}+n^{4}\right)+2\left(B_{2}+B_{5}\right) m_{1}^{2} n^{2}}\right\}+K_{w}+K_{p}\left(m_{1}^{2}+n^{2}\right) / R^{2}
\end{array}\right\}}
$$

of FGM material properties can be expressed as

$$
P=P_{0}\left(P_{-1} T^{-1}+1+P_{1} T+P_{2} T^{2}+P_{3} T^{3}\right)
$$

in which $T=300 \mathrm{~K}$ (room temperature), $P_{0}, P_{-1}, P_{1}, P_{2}$, $P_{3}$ are the coefficients of temperature $T(\mathrm{~K})$ expressed in Kelvin and are unique to the constituent materials Reddy and Chin [4].

Typical values for $\mathrm{ZrO}_{2}$ and $\mathrm{Ti}-6 \mathrm{Al}-4 \mathrm{~V}$ listed in Table 3. The materials are assumed to be perfectly elastic throughout the deformation. $E_{0 c}, E_{0 m}, v_{0 c}, v_{0 m}$, are the Young's modulus and Poisson's ratio of the metal and ceramic materials of the cylindrical shell, respectively.

Numerical computations, for pure metal, pure ceramic and three layered cylindrical shells containing an FGM layer with or without the Pasternak-Winkler foundations have been carried out using expression (20). The results are presented in Table 4 and Figures 4-7. The results given in all tables below for the values of the critical axial load, corresponding to the numbers of longitudinal and circumferential waves $(m, n)$ are presented in parentheses.

In Table 4 variations of the critical axial load and corresponding wave numbers for pure metal, pure ceramic and three layered cylindrical shells containing an FGM layer with different compositional profiles $(N=0.5 ; 1 ; 2$; $15)$, versus the Winkler foundation stiffness $K_{w}$ and the shear subgrade modulus $K_{p}$ are presented. It is observed that the critical axial load of three layered cylindrical shells containing an FGM layer increases gradually with increasing $K_{w}$ or $K_{p}$ separately or together for all compositional profiles. The effect of the composi ional profiles on the critical axial loads reduce, as the considering affect of elastic foundations. Further more, for the small values of $K_{w}$ and $K_{p}$ the variations of volume fractions of the FGM has a considerable influence on the values of the critical axial load. When the volume fraction index $N$ increases, the values of the critical axial loads with and without elastic foundation decrease. When the volume fraction index $N$ increases, the effect to the critical axial loads with and without elastic foundation increases.

When the shear subgrade modulus $K_{p}$ is zero and the Winkler foundation stiffness $K_{w}$ is changed, the effect on the critical axial load $\left(T_{w}^{c r}\right)$ are $5.95 \%, 11.62 \% ; 49.08 \%$ for $K_{w}=5 \times 10^{7} ; 10^{8} ; 5 \times 10^{8}\left(\mathrm{~N} / \mathrm{m}^{3}\right)$, respectively, for $N=$ 0.5 of the FGM layer of three layered cylindrical shells.

When $K_{p}$ is keep constant $\left(K_{p}=10^{6} \mathrm{~N} / \mathrm{m}\right)$ and the Winkler foundation stiffness $K_{w}$ is changed the effect on the critical axial load $\left(T_{w p}^{c r}\right)$ are $25.82 \%, 31.48 \% ; 69 \%$ for $K_{w}=5 \times 10^{7} ; 10^{8} ; 5 \times 10^{8}\left(\mathrm{~N} / \mathrm{m}^{3}\right)$, respectively, for $N=$ 0.5 of the FGM layer of three layered cylindrical shells.

\begin{tabular}{|c|c|c|c|c|}
\hline \multirow[t]{2}{*}{ Coefficients } & \multicolumn{2}{|c|}{ Zirconia $\left(\mathrm{ZrO}_{2}\right)$} & \multicolumn{2}{|c|}{ Titanium alloy (Ti-6Al-4V) } \\
\hline & $\mathrm{E}_{\mathrm{c}}(\mathrm{Pa})$ & $v_{c}$ & $\mathrm{E}_{\mathrm{m}}(\mathrm{Pa})$ & $v_{m}$ \\
\hline $\mathrm{P}_{0}$ & $2.4427 \times 10^{11}$ & 0.2882 & $1.2256 \times 10^{11}$ & 0.2884 \\
\hline$P_{-1}$ & 0 & 0 & 0 & 0 \\
\hline$P_{1}$ & $-1.371 \times 10^{-3}$ & $1.133 \times 10^{-4}$ & $-4.586 \times 10^{-4}$ & $-1.121 \times 10^{-4}$ \\
\hline $\mathrm{P}_{2}$ & $1.214 \times 10^{-6}$ & 0 & 0 & 0 \\
\hline $\mathrm{P}_{3}$ & $-3.681 \times 10^{-10}$ & 0 & 0 & 0 \\
\hline$P$ & $1.68063 \times 10^{11}$ & 0.2980 & $1.05698 \times 10^{11}$ & 0.2981 \\
\hline
\end{tabular}

Table 3. Temperature-dependent coefficients of Young's modulus E (MPa) and Poisson's ratio $v$ for ceramics and metals (Reddy and Chin [4]). 
Table 4. Variations of critical axial loads and corresponding circumferential wave numbers for pure metal, pure ceramic and three layered FGM cylindrical shells versus the foundations moduli $K_{w}$ and $K_{p}\left(h / h_{1}=h / h_{2}=2, h / a=4, L / R=3 ; R / h=200\right)$.

\begin{tabular}{|c|c|c|c|c|c|c|c|}
\hline \multirow{2}{*}{$K_{w}$} & \multirow{2}{*}{$K_{P}$} & \multirow[b]{2}{*}{$\mathrm{ZrO}_{2}$} & \multicolumn{4}{|c|}{$\mathrm{ZrO}_{2}-\mathrm{FGM}-\mathrm{Ti}-6 \mathrm{Al}-4 \mathrm{~V}$} & \multirow{2}{*}{$T i-6 \mathrm{Al}-4 \mathrm{~V}$} \\
\hline & & & $N=0.5$ & $N=1$ & $N=2$ & $N=15$ & \\
\hline \multirow[t]{2}{*}{0} & 0 & $7.364(24,4)$ & $5.062(6,11)$ & $4.969(16,12)$ & $4.873(22,7)$ & $4.684(19,10)$ & $3.695(24,4)$ \\
\hline & 0 & $7.663(25,2)$ & $5.363(25,2)$ & $5.275(25,2)$ & $5.184(25,2)$ & $5.001(25,2)$ & $3.990(25,2)$ \\
\hline \multirow{2}{*}{$5 \times 10^{7}$} & $10^{6}$ & $8.669(25,2)$ & $6.369(25,2)$ & $6.281(25,2)$ & $6.190(25,2)$ & $6.007(25,2)$ & $4.996(25,2)$ \\
\hline & 0 & $7.955(25,2)$ & $5.650(25,2)$ & $5.566(26,2)$ & $5.475(25,2)$ & $5.293(25,2)$ & $4.262(26,2)$ \\
\hline \multirow[t]{3}{*}{$1 \times 10^{8}$} & $10^{5}$ & $8.055(25,2)$ & $5.751(26,2)$ & $5.667(26,2)$ & $5.576(25,2)$ & $5.393(25,2)$ & $4.363(26,2)$ \\
\hline & $10^{6}$ & $8.961(25,2)$ & $6.656(26,2)$ & $6.572(26,2)$ & $6.481(25,2)$ & $6.298(25,2)$ & $5.267(26,2)$ \\
\hline & 0 & $9.957(28,2)$ & $7.547(30,2)$ & $7.475(30,2)$ & $7.399(30,2)$ & $7.226(30,2)$ & $6.009(31,2)$ \\
\hline \multirow[t]{2}{*}{$5 \times 10^{8}$} & $10^{5}$ & $10.058(28,2)$ & $7.647(30,2)$ & $7.575(30,2)$ & $7.500(30,2)$ & $7.327(30,2)$ & $6.110(31,2)$ \\
\hline & $10^{6}$ & $10.962(29,2)$ & $8.551(30,2)$ & $8.479(30,2)$ & $8.403(30,2)$ & $8.230(30,2)$ & $7.013(31,2)$ \\
\hline
\end{tabular}

When $K_{w}$ is keep constant $\left(K_{w}=10^{8} \mathrm{~N} / \mathrm{m}^{3}\right)$ and the shear subgrade modulus $K_{p}$ is changed the effect on the critical axial load $\left(T_{w p}^{c r}\right)$ are $13.61 \%, 31.48 \%$ for $K_{p}=10^{5} ; 10^{6}(\mathrm{~N} / \mathrm{m})$, respectively, for $N=0.5$ of the FGM layer of three layered cylindrical shells.

Note, the following expression is used for percents:

$$
\left[\left(T_{w p}^{c r}-T^{c r}\right) / T^{c r}\right] \times 100 \%
$$

In Figure 3 shows the variations of the values of critical axial loads for the cylindrical shell composed of $\mathrm{ZrO}_{2}-F G M-T i-6 \mathrm{Al}-4 \mathrm{~V}$ layers with and without elastic foundation with respect to $h / 2 a$. When $h / 2 a=1$, the three layered cylindrical shell containing an FGM layer transformed to the pure FGM cylindrical shell.

When the ratio, $h / 2 a$, increases, the values of the critical axial loads for the composite cylindrical shells composed of $\mathrm{ZrO}_{2}-F G M-T i-6 \mathrm{Al}-4 \mathrm{~V}$ layers with and without elastic foundations increase. On the other hand, when FGM layer thickness is increased, the values of the critical axial loads with and without elastic foundation for the three layered cylindrical shells degrease.

Figure 4 shows the variations of the values of critical axial loads for the composite cylindrical shell composed of $\mathrm{ZrO}_{2}-F G M-T i-6 \mathrm{Al}-4 \mathrm{~V}$ layers with respect to $h / 2 a$.

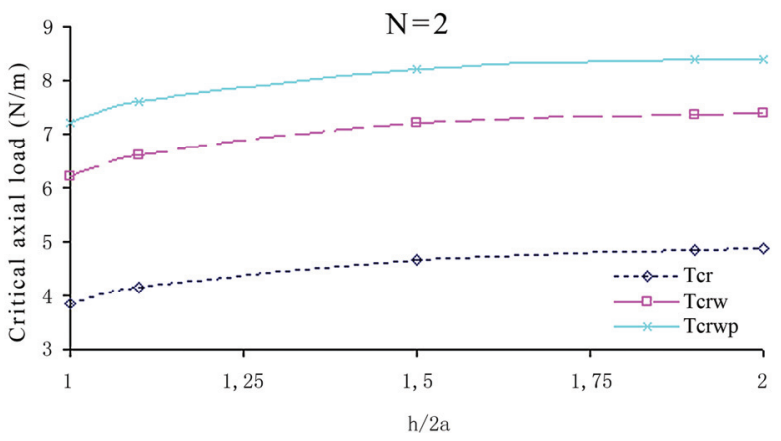

Figure 3. Variations of critical axial loads for three layered FGM cylindrical shells with and without elastic foundations for $N=2$, versus $h / 2 a\left(h_{1}=h_{2}, R / h=200, L / R=3 ; K_{w}=\right.$ $\left.5 \times 10^{8} \mathrm{~N} / \mathrm{m}^{3} ; \mathrm{K}_{\mathrm{p}}=10^{6} \mathrm{~N} / \mathrm{m}\right)$.

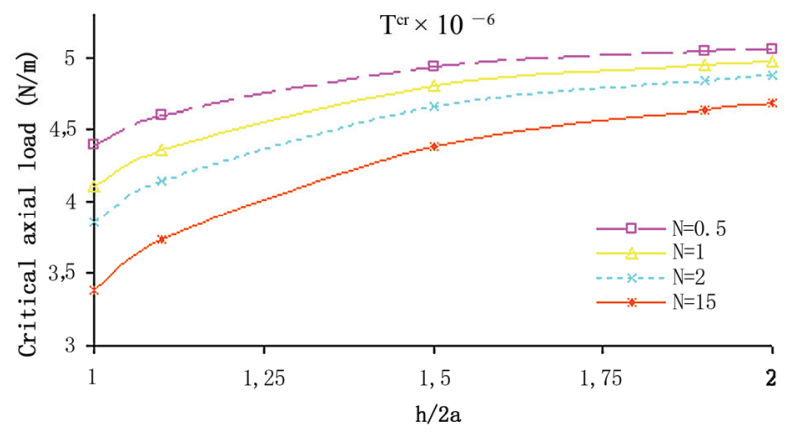

Figure 4. Variations of critical axial loads for three layered FGM cylindrical shells without elastic foundations versus $h / 2 a ;\left(h_{1}=h_{2} ; R / h=200 ; L / R=3\right)$.

The effects of compositional profiles on the critical axial load of three layered cylindrical shells containing an FGM layer are decreased, as the ratio $h / 2 a$ increase. For example, comparing the values of the critical axial load of three layered cylindrical shells containing an FGM layer with the values for pure ceramic cylindrical shells, at $N=2 ; h / 2 a=1 ; 1.5 ; 2.0$, the effects are $47.7 \%$; $36.7 \% ; 33.8 \%$ as $K_{w}=5 \times 10^{8} \mathrm{~N} / \mathrm{m}^{3}, K_{p}=0$, respectively.

Figure 5 shows the variations of the values of critical axial loads for the composite cylindrical shell composed of ZrO2-FGM-Ti-6Al-4V layers on a Winkler foundation with respect to $h / 2 a$. The effects of compositional profiles on the critical axial load of three layered cylindrical shells containing an FGM layer resting on a Winkler foundation are decreased, as the ratio $h / 2 a$ increase. For example, comparing the values of the critical axial loads of three layered cylindrical shells containing an FGM layer with the values for pure ceramic cylindrical shells, at $\mathrm{N}=2 ; \mathrm{h} / 2 \mathrm{a}=1 ; 1.5 ; 2.0$, the effects are $37.8 \%$; $27.6 \% ; 25.7 \%$ as $K_{w}=5 \times 10^{8} \mathrm{~N} / \mathrm{m}^{3}, K_{p}=0$, respectively.

Figure 6 shows the variations of the values of critical axial loads for the composite cylindrical shell composed of $\mathrm{ZrO}_{2}-\mathrm{FGM}$-Ti-6Al-4V layers on a Pasternak foundation with respect to $h / 2 a$ ratios. As the ratio $h / 2 a$ increase, the effects of compositional profiles on the critical axial 
load of three layered cylindrical shells containing an FGM layer resting on a Winkler foundation are decreased. For example, comparing the values of the critical axial loads of three layered cylindrical shells containing an FGM layer with the values for pure ceramic cylindrical shells, at $N=2 ; h / 2 a=1 ; 1.5 ; 2.0$, the effects are $34.3 \%$; $25.1 \% ; 23.3 \%$ as $K_{w}=5 \times 10^{8} \mathrm{~N} / \mathrm{m}^{3}, \quad K_{p}=10^{6} \mathrm{~N} / \mathrm{m}$, respectively.

In Figure 7 are illustrated variations of critical axial loads for three layered FGM cylindrical shells with and without elastic foundation versus $R / h$. When the ratio $R / h$ increases, the values of the critical axial loads for cylindrical shells composed of $\mathrm{ZrO}_{2}-F G M-T i-6 \mathrm{Al}-4 \mathrm{~V}$ layers with and without elastic foundation decrease. The effect of the elastic foundation on the values of the critical axial load of three layered cylindrical shells containing an FGM layer are increased, as the ratio $R / h$ increase. For example, comparing the values of the critical axial load of three layered cylindrical shells containing an FGM layer with the values for pure ceramic cylindrical shells, at $\mathrm{N}=2 ; R / h=100 ; 200 ; 300$, the effects are $15.34 \% ; 51.8 \% ; 91.1 \%$ as $K_{w}=5 \times 10^{8} \mathrm{~N} / \mathrm{m}^{3}, K_{p}=0,25.7 \%$; $72.4 \% ; 128.9 \%$ as $K_{w}=5 \times 10^{8} \mathrm{~N} / \mathrm{m}^{3}, K_{p}=10^{6} \mathrm{~N} / \mathrm{m}$ respectively.

\section{Conclusions}

In this study, the stability of cylindrical shells that composed of ceramic, FGM, and metal layers subjected to axial load and resting on a Pasternak foundation is investigated. Material properties of an FGM layer are varied continuously in thickness direction according to a simple power distribution in terms of the ceramic and metal volume fractions. The modified Donnell type stability and compatibility equations on a Pasternak foundation are obtained. Applying Galerkin's method analytic solutions are obtained for the critical axial load of three-

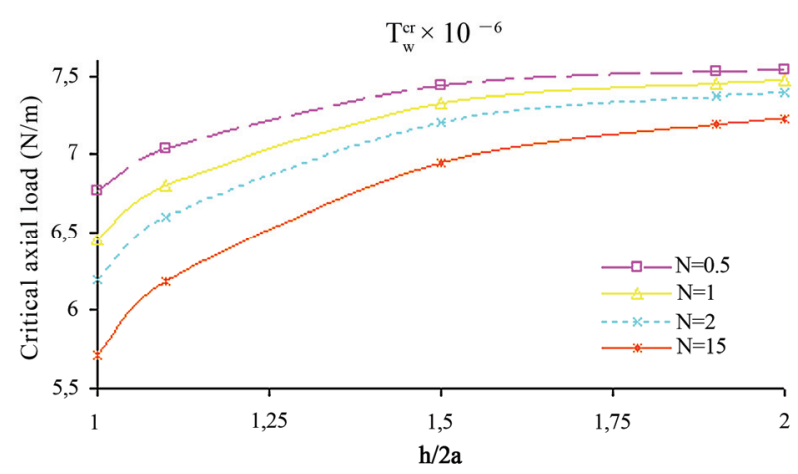

Figure 5. Variations of critical axial loads for three layered FGM cylindrical shells on a Winkler foundation versus $\mathbf{h} / \mathbf{2 a}$ $\left(h_{1}=h_{2} ; R / h=200 ; L / R=3 ; K_{w}=5 \times 10^{8} \mathrm{~N} / \mathrm{m}^{3}\right)$.

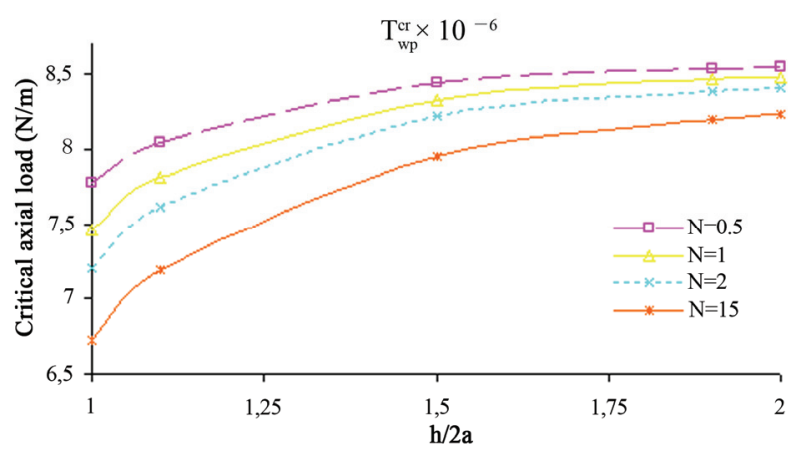

Figure 6. Variations of critical axial loads for three layered FGM cylindrical shells on a Pasternak foundation versus $\mathbf{h} / \mathbf{2 a}$ $\left(h_{1}=h_{2} ; R / h=200 ; L / R=3 ; K_{w}=5 \times 10^{8} \mathrm{~N} / \mathrm{m}^{3} ; K_{p}=10^{6} \mathrm{~N} / \mathrm{m}\right)$.

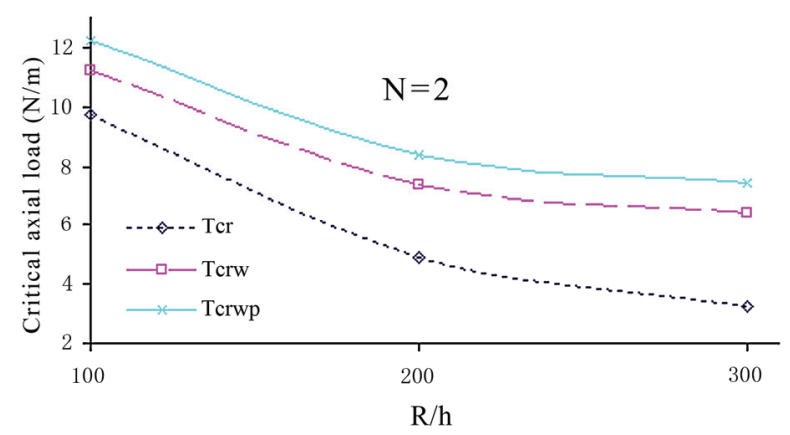

Figure 7. Variations of critical axial loads for three layered FGM cylindrical shells with and without elastic foundations versus $R / h\left(h_{1}=h_{2} ; L / R=3 ; K_{w}=5 \times 10^{8} \mathrm{~N} / \mathrm{m}^{3} ; K_{p}=10^{6} \mathrm{~N} / \mathrm{m}\right)$.

layered cylindrical shells containing an FGM layer with and without elastic foundations. The detailed parametric studies are carried out to study the influences of thickness variations of the FGM layer, radius-to-thickness ratio, material composition and material profile index, Winkler and Pasternak foundations on the critical axial load of three-layered cylindrical shells. Comparing results with those in the literature validates the present analysis.

\section{Acknowledgements}

This study is supported by the Scientific and Technical Research Council of Turkey (TUBITAK) under Project Number 108M322. The authors thank to TUBITAK for the support of the project.

\section{References}

[1] M. Koizumi, "The Concept of FGM," Ceramic Transactions, Functionally Gradient Materials, Vol. 34, 1993, pp. 3-10.

[2] B. Kieback, A. Neubrand and H. Riedel, "Processing techniques for Functionally Graded Materials," Mate- 
rials Science and Engineering A, Structural Materials: Properties, Microstructure and Processing, Vol. 362, No. 1-2, 2003, pp. 81-105.

[3] V. Birman, "Buckling of Functionally Graded Hybrid Composite Plates," Proceedings of the 10th Conference on Engineering Mechanics, Boulder, USA, 1995.

[4] J. N. Reddy and C. D. Chin, "Thermomechanical Analysis of Functionally Graded Cylinders and Plates," Journal of Thermal Stresses, Vol. 21, 1998, pp. 593-602.

[5] T. Y. Ng, K. Y. Lam, K. M. Liew and J. N. Reddy, "Dynamic Stability Analysis of Functionally Graded Cylindrical Shells under Periodic Axial Loading," International Journal of Solids Structures, Vol. 38, No. 8, 2001, pp. 1295-1309.

[6] A. H. Sofiyev, "Dynamic Buckling of Functionally Graded Cylindrical Shells under Non-periodic Impulsive Loading," Acta Mechanica, Vol. 165, No. 3-4, 2003, pp. 153-162.

[7] H. S. Shen and N. Noda, "Post-buckling of FGM Cylindrical Shell under Combined Axial and Radial Mechanical Loads in Thermal Environments," International Journal of Solids Structures, Vol. 42, No. 16, 2005, pp. 4641- 4662.

[8] H. S. Shen, "Functionally Graded Materials: Nonlinear Analysis of Plates and Shells," CRC Press, Boca Raton, 2009.

[9] S. Pitakthapanaphong and E. P. Busso, "Self-consistent elasto-plastic Stress Solutions for Functionally Graded Material Systems Subjected to Thermal Transients," Journal of Mechanics and Physics of Solids, Vol. 50, No. 4, 2002, pp. 695-716.

[10] K. S. Na and J. H. Kim, "Three-dimensional Thermomechanical Buckling Analysis for Functionally Graded Composite Plates," Composite Structures, Vol. 73, No. 4, 2006, pp. 413-422.

[11] K. M. Liew, J. Yang and Y. F. Wu, "Nonlinear Vibration of a Coating-FGM-substrate Cylindrical Panel Subjected to a Temperature Gradient," Computer Methods in Applied Mechanics and Engineering, Vol. 195, No. 9-12, 2006, pp. 1007-1026.

[12] S. R. Lia and R. C. Batra, "Buckling of Axially Compressed Thin Cylindrical Shells with Functionally Graded Middle Layer," Thin Walled Structures, Vol. 44, No. 10, 2006, pp. 1039-1047.

[13] A. H. Sofiyev, A. Deniz, I. H. Akcay and E. Yusufoglu, "The Vibration and Stability of a Three-layered Conical Shell Containing a FGM Layer Subjected to Axial Com- pressive Load," Acta Mechanica, Vol. 183, No. 3-4, 2006, pp. 129-144.

[14] A. H. Sofiyev, "Vibration and Stability of Composite Cylindrical Shells Containing a FG Layer Subjected to Various Loads," Structural Engineering and Mechanics an International Journal, Vol. 27, No. 3, 2007, pp. 365391.

[15] P. L. Pasternak, "On a New Method of Analysis of an Elastic Foundation by Means of Two Foundation Constants," Gosudarstvennoe Izdatelstvo Literaturi po Stroitelstvu I Arkhitekture, Moscow, 1954.

[16] V. A. Bajenov, "The Bending of the Cylindrical Shells in an Elastic Medium," Visha Shkola, Kiev, 1975.

[17] D. N. Paliwal, R. K. Pandey and T. Nath, "Free Vibration of Circular Cylindrical Shell on Winkler and Pasternak Foundation," International Journal of Pressure Vessels and Piping, Vol. 69, 1996, pp. 79-89.

[18] T. Y. Ng and K. Y. Lam, "Free Vibrations Analysis of Rotating Circular Cylindrical Shells on an Elastic Foundation," Journal of Vibration and Acoustics, Vol. 122, No. 1, 2000, pp. 85-89.

[19] H. G. Tj, T. Mikami, S. Kanie and M. Sato, "Free Vibration Characteristics of Cylindrical Shells Partially Buried in Elastic Foundations," Journal of Sound and Vibration, Vol. 290, No. 3, 2006, pp. 785-793.

[20] A. H. Sofiyev, S. N. Keskin and Al. H. Sofiyev, "Effects of Elastic Foundation on the Vibration of Laminated Nonhomogeneous Orthotropic Circular Cylindrical Shells," Journal of Shock and Vibration, Vol. 11, 2004, pp. 89-101.

[21] Z. Q. Cheng and S. Kitipornchai, "Membrane Analogy of Buckling and Vibration of Inhomogeneous Plates," Journal of Engineering Mechanics-ASCE, Vol. 125, No. 11, 1999, pp. 1293-1297.

[22] Z. Y. Huang, C. F. Lu and W. Q. Chen, "Benchmark Solutions for Functionally Graded Thick Plates Resting on Winkler-Pasternak Elastic Foundations," Composite Structures, Vol. 85, No. 2, 2008, pp. 95-104.

[23] P. Malekzadeh, "Three-dimensional Free Vibration Analysis of Thick Functionally Graded Plates on Elastic Foundations," Composite Structures, Vol. 89, No. 3, 2009, pp. 367-373.

[24] V. L. Agamirov, "Dynamic problems of nonlinear shells theory", Nauka, Moscow, 1990.

[25] R. M. Jones and H. S. Morgan, "Buckling and Vibration of Cross-ply Laminated Circular Cylindrical Shells," American Institute of Aeronautics and Astronautics Journal, Vol. 13, No. 5, 1975, pp. 664-671. 